\title{
Viral and Psychiatric Disorders in Methadone Maintenance Therapy (MMT) Clients
}

\author{
Hamid Abousaeedi, ${ }^{1}$ Omid Reza Hosseini, ${ }^{2,}$ Reza Bidaki, ${ }^{3}$ Mahmood Sheikh Fathollahi, ${ }^{4}$ Hamid Reza \\ Goodarzi Nejad, ${ }^{5}$ Golam Hossein Hassan Shahi, ${ }^{6}$ Maryam Sahraei, ${ }^{7}$ Shila Jalalpoor, ${ }^{8}$ and Mina
}

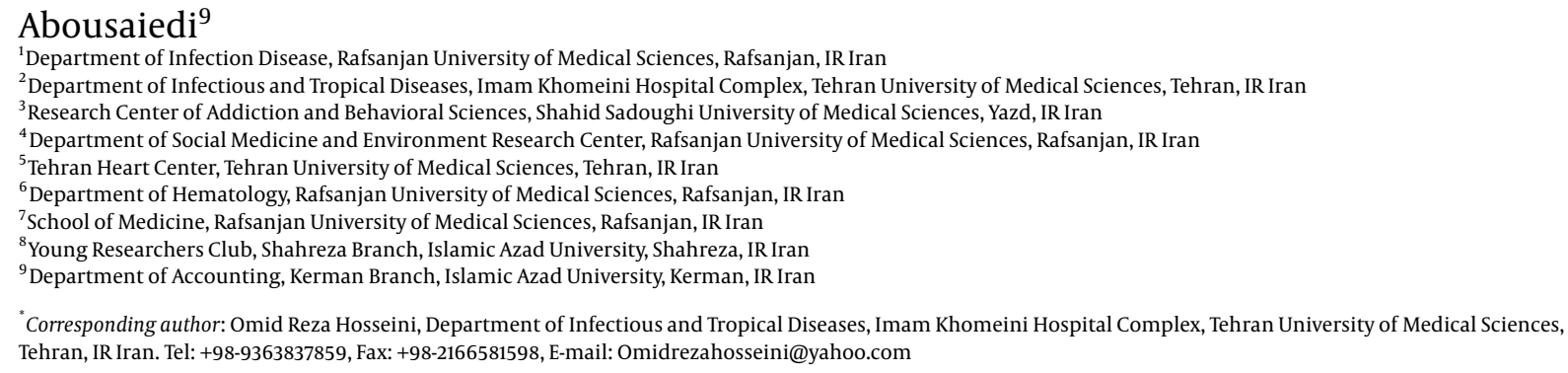

Received 2016 January 15; Revised 2016 February 06; Accepted 2016 February 29.

\begin{abstract}
Background: Methadone maintenance therapy (MMT) is currently widely accepted as the treatment of choice for opioid replacement therapy.

Objectives: The aim of this study was to determine the prevalence of HBV, HCV, and HIV infections as well as psychiatric disorders in our MMT cases.

Patients and Methods: Using a cross-sectional study design, we studied patients treated for opioid abuse or addiction who attended MMT programs in Rafsanjan, Iran, from March to June 2012. HIV, HBV, and HCV test reports were recorded from MMT clients' documents (ELISA and Western blotting results), and psychiatric disorders were diagnosed by two trained psychology interviewers according to the DSM-IV criteria.

Results: Of the 1,825 patients included in this study, 1,737 (95.2\%) were men with a mean age of $28.00 \pm 7.69$ years (range: 18 - 52 years). In total, 64 (3.5\%) subjects showed symptoms of depression and $21(1.2 \%)$ had anxiety. The prevalence of HBV, HCV, and HIV were 4 (0.2\%), 31 (1.7\%), and 18 (1.0\%), respectively. The association between psychiatric disorders and chronic diseases did not reach a statistically significant level (Fisher's exact test, $\mathrm{P}=0.193$ ).

Conclusions: The prevalence of HBV, HIV, and psychiatric disorders in our study group was lower than in previous reports. This may be because we entered participants from private MMT centers. Therefore, our results are not generalizable to other populations. Nevertheless, HCV prevalence was higher, and our results lead us to recommend that training programs be planned to prevent the outbreak of these viral infections.
\end{abstract}

Keywords: Methadone, HBV, HCV, HIV, Depression, Anxiety

\section{Background}

Narcotic addiction is a serious health problem in communities with economic, social, and hygienic challenges (1). A large proportion of viral infections, particularly hepatitis B virus (HBV), hepatitis C virus (HCV), and human immunodeficiency virus (HIV) are prevalent among drug addicts. Drug abuse is one of the main public health issues in Iran and neighboring countries. According to reports of drug abuse in Iran, approximately 1.2 million people are in need of treatment services (2). Currently, intravenous (IV) drug abuse is the most prominent risk factor for transmission of HBV, HCV, and HIV (3-6). Overall, psychi- atric disorders are more prevalent among drug addicted individuals (7). The diagnosis of these disorders plays a decisive role in the choice of medication and in the patient's prognosis. Iranian psychiatrists and other professionals in this field have used different methods of treatment to overcome this problem (8). Methadone, as a substitution therapy for interrupting opiate addiction, was first used in North America. At present, methadone maintenance therapy (MMT) is widely accepted as a choice for opioid replacement therapy worldwide (9). Patients with high levels of emotional distress and psychiatric disorders should be maintained on higher daily doses of methadone. How- 
ever, some groups, such as those with HIV/AIDS and hepatitis infections, should be maintained on lower daily doses of methadone (10-12). Therefore, it is necessary to determine the presence of such conditions before the initiation of MMT.

\section{Objectives}

The present study evaluated the prevalence of HIV, HBV, and HCV infections along with specific psychiatric disorders in patients referred to the MMT clinics in Rafsanjan, Iran in 2012.

\section{Patients and Methods}

In the current cross-sectional study, we targeted the population of clients who were treated for opiate abuse and attended maintenance programs in Rafsanjan, Iran during 2012. There are 15 private MMT clinics in Rafsanjan, so we used a stratified random cluster sampling proportional to the number of clients covered by each of the 15 private clinics. The sample size was measured by the frequency of viral and psychiatric disorders in previous studies. The aim of this study was initially explained to the clinic directors, and their consent was obtained for participation in the study. The diagnostic and statistical manual of mental disorders IV (DSM IV) criteria was used for opioid dependence establishment (12). Interviews were carried out by two trained interviewers from March to June 2012.

Demographic data (e.g., age, sex, years of education, marital status, and career status), the addiction durations, urinary morphine tests, and the patients' MMT histories (previous methadone treatments and the patients' daily doses of received methadone) were also collected by a questionnaire.

A written consent form was also obtained from each client and the study protocol was approved by the Rafsanjan University of Medical Sciences ethical committee. Psychiatric disorders including sleep disorders, phobias, obsessive compulsive disorder (OCD), schizophrenia, and post-traumatic stress disorder (PTSD) were screened according to the DSM-IV axis I disorders: clinician version (13). HIV, HBV, and HCV test reports (enzyme-linked immunosorbent assay (ELISA) and Western blotting) were recorded in the MMT clients' documents and were the source of data. HbsAg, HBsAb, HCVAb, HIVAb, and Western blot tests were recorded.

Other chronic diseases including diabetes mellitus, hypertension, hyperlipidemia, ischemic heart diseases, renal failure, chronic lung diseases, or other diseases in clients' documents. These diseases were selected because they are the most frequent chronic diseases in humans. Data were registered in an anonymous form.

Data were analyzed statistically by the statistical package for the social sciences (SPSS 18.0), and Chi-square or independent two-sample t-tests were applied. The level of significance was considered at $\mathrm{P} \leq 0.05$.

\section{Results}

Of the 1,825 patients who referred to MMT clinics for treatment of opiate addiction, 1,737 (95.2\%) were men with a mean age of $28.00 \pm 7.69$ years (mean \pm SD; range: 18 - 52 years).

The relationships between psychotic disorders and other evaluated variables are shown in Table1. The relationships between chronic diseases and other evaluated variables are depicted in Table 2.

Urinary morphine tests were positive in 224/1,724 (13.0\%, 95\% CI: $11.4 \%-14.6 \%)$ of subjects. The frequency of drug withdrawal was $2.60 \pm 2.21$ times (range: 0 - 11 times). The mean dose of prescribed methadone was 70.49 \pm 43.53 mg (range: 12 - $320 \mathrm{mg}$ ).

In total, 64 (3.5\%, 95\% CI: 2.7\% - 4.3\%) patients showed symptoms of depression, 21 (1.2\%, 95\% CI: 0.7\% - 1.7\%) had anxiety, and 59 (3.2\%, 95\% CI: $2.4 \%-4.0 \%)$ had other psychiatric disorders including sleep disorders, phobias, OCD, schizophrenia, and PTSD.

The prevalence of HBV, HCV, and HIV were 4 (0.2\%, 95\% CI: $0 \%-0.4 \%), 31(1.7 \%, 95 \% \mathrm{CI}: 1.1 \%-2.3 \%)$, and 18 (1.0\%, 95\% CI: $0.5 \%-1.5 \%)$, respectively.

The association between psychotic disorders and chronic diseases did not reach a statistically significant level (Fisher's exact test, $\mathrm{P}=0.193$ ).

\section{Discussion}

According to the most recent reports up to 2007, approximately two million HBV carriers are living in Iran, which is approximately $3 \%$ of the Iranian population (14). However, in our study, the prevalence of HBV was $0.2 \%$, while the prevalence of HCV in the MMT cases in Rafsanjan was $1.7 \%$. According to the latest reports reviewed by Alavian (15) in 2009, the HCV prevalence in the general population of Iran is $0 \%-1.3 \%$.

The prevalence of HIV in our study was $1.0 \%$, which was higher than that speculated $(0.16 \%)$ in the Iranian population (16). Most of the cases in our study had a history of more than one attempt at drug withdrawal. This can be related to various factors that interfere with successful drug 
Table 1. Association Between Psychotic Disorders and Other Evaluated Variables ${ }^{\mathrm{a}}$

\begin{tabular}{|c|c|c|c|c|c|}
\hline Variable & No. Disease $(n=1,681)$ & Depression $(n=64)$ & $\operatorname{Anxiety}(n=21)$ & Other Diseases $(n=59)$ & PValue $^{\mathrm{b}}$ \\
\hline \multicolumn{6}{|l|}{ Sex } \\
\hline Male & $1,607(92.5)$ & $54(3.1)$ & $21(1.2)$ & $55(3.2)$ & 0.003 \\
\hline \multicolumn{6}{|l|}{ Job } \\
\hline Employed & $1,370(92.9)$ & $39(2.6)$ & $16(1.1)$ & $50(3.4)$ & 0.001 \\
\hline \multicolumn{6}{|l|}{ Marriage status } \\
\hline Married & $1,494(93.0)$ & $50(3.1)$ & $17(1.1)$ & $46(2.9)$ & 0.003 \\
\hline \multicolumn{6}{|l|}{ Morphine test $^{c}$} \\
\hline Positive & $199 / 224(88.8)$ & $9 / 224(4.0)$ & $0 / 224(0)$ & $16 / 224(7.1)$ & 0.002 \\
\hline Age, $y$ & $26(23-30)$ & $25(23.25-30)$ & $24(21-27.50)$ & $26(23-29)$ & 0.248 \\
\hline Methadone dose, $\mathrm{mg}^{\mathrm{d}}$ & $60(48-76)$ & $60(48-80)$ & $50(42-60)$ & $56(48-65)$ & 0.024 \\
\hline No. of withdrawals & $2(1-4)$ & $2.50(1-4)$ & $2(1-3.50)$ & $2(1-4)$ & 0.169 \\
\hline \multicolumn{6}{|c|}{$\begin{array}{l}{ }^{\text {a }} \text { Data are expressed as No. (\%) or median (1st quartile-3rd quartile). } \\
{ }^{b} \text { P values are significant at } \leq 0.05 \text { level, } 2 \text {-sided. } \\
{ }^{\mathrm{c}} \text { There were some missing data in morphine test variables. } \\
{ }^{\mathrm{d}} \text { There were no significant differences in multiple comparisons performed using Mann-Whitney U tests when applying Bonferroni corrections at an } \alpha=0.05 \text { level. }\end{array}$} \\
\hline
\end{tabular}

Table 2. Association Between Chronic Diseases and Other Evaluated Variables ${ }^{\mathrm{a}}$

\begin{tabular}{|c|c|c|c|c|}
\hline Variable & No. Disease $(n=1,725)$ & HBV, HCV, HIV $(n=53)$ & Other Diseases $(\mathbf{n}=\mathbf{2 0})$ & PValue $^{b}$ \\
\hline \multicolumn{5}{|l|}{ Sex } \\
\hline Male & $1,669(96.1)$ & $50(2.9)$ & $18(1.0)$ & 0.337 \\
\hline \multicolumn{5}{|l|}{ Job } \\
\hline Employed & $1,433(97.2)$ & $28(1.9)$ & $14(0.9)$ & $<0.001$ \\
\hline \multicolumn{5}{|l|}{ Marriage status } \\
\hline Married & $1,561(97.1)$ & $31(1.9)$ & $15(0.9)$ & $<0.001$ \\
\hline \multicolumn{5}{|l|}{ Morphine test ${ }^{c}$} \\
\hline Positive & $198 / 224(88.4)$ & $24 / 224(10.7)$ & $2 / 224(0.9)$ & $<0.001$ \\
\hline Age, $y$ & $27.95 \pm 7.67$ & $28.49 \pm 7.93$ & $30.45 \pm 8.75$ & 0.315 \\
\hline Methadone dose, mgd & $69.38 \pm 43.14^{d}$ & $107.60 \pm 46.46^{d}$ & $69.50 \pm 22.12^{d}$ & $<0.001$ \\
\hline No. of withdrawals & $2.56 \pm 2.19^{\mathrm{d}}$ & $3.79 \pm 2.35^{\mathrm{d}}$ & $2.85 \pm 2.62^{\mathrm{d}}$ & $<0.001$ \\
\hline
\end{tabular}

${ }^{\mathrm{a}}$ Data are expressed as No. (\%) or mean $\pm \mathrm{SD}$ (standard deviation).

${ }^{\mathrm{b}} \mathrm{P}$ values are significant at $\leq 0.05$ level, 2 -sided.

${ }^{\mathrm{c}}$ There were some missing data in morphine test variables.

${ }^{\mathrm{d}}$ Means with different superscript letters are significantly different from each other, when performing Tukey's multiple comparisons test.

withdrawals, including background diseases such as depression, or stimulant environmental factors such as addicted friends. Moreover, $13 \%$ of cases had positive morphine tests. This suggests that some of our cases were simultaneously abusing opioids while on methadone therapy. This may be due to insufficient prescribed methadone doses. In the MMT methods, most cases require $60-120$ mg of methadone per day, but it is suggested that people with a high level of emotional distress, cluster A, or schizophrenia-like personality disorders should be maintained on higher daily doses of methadone (12).

In our study, no relationships were observed between sex and age with psychiatric disorders. Cases with psychiatric disorders were more likely to be employed and married, as well as their morphine test is less positive. This may indicate that individuals with psychiatric disorders (who are married or employed) had fewer tendencies to abuse drugs during their enrollment in the MMT 
program. We found that the mean dose of prescribed methadone was higher in patients with HBV, HCV, and HIV, and viral-infected patients had more withdrawal attempts compared to patients who lacked coinfections. According to a study by Golden and colleagues, depression and anxiety had a higher prevalence in HCV, especially among cases entering MMT programs (13); however, we observed no such relationship between psychiatric disorders and viral infections.

In our study, the prevalence of psychiatric disorders such as depression (1.2\%) and anxiety (3.2\%) was lower than in previous reports $(15,16)$. In contrast to our findings, a study performed in Kerman (geographically close to Rafsanjan) reported $24.7 \%$ of MMT referrals were simultaneously suffering from major depressive disorders including bipolar mood disorder (4\%) as well as simultaneous major depressive disorder and anxiety (11\%) (17). Moreover, in a study by Shirazi et al. (18), $68.6 \%$ of addicted patients had a history of depression.

In the present study, psychiatric disorders were more prevalent in males, employed, and married patients, while individuals with positive morphine tests showed a lower incidence of psychiatric disorders. There was no relationship between psychiatric disorders with age, methadone dosage, and withdrawal attempts. In a study by Parvaresh et al. (17), significant correlations were observed between increased methadone dosage and an antisocial personality. Furthermore, it was suggested that high methadone doses can be a marker of simultaneous psychiatric disorders in patients that should be assessed.

One of our study limitations was that we could not assess the frequency of occult hepatitis in our population because the supplementary laboratory test results were not available in the clients' documents. Moreover, we only had private MMT clinics in our city. The accuracy of the recorded medical histories, especially concerning chronic diseases, was another limitation.

In conclusion, the prevalence of HBV, HIV, and psychiatric disorders in MMT cases in Rafsanjan was lower than in previous reports. This may be because we entered participants from private MMT centers who may have better socioeconomic statuses (suggested by their ability to afford the expenses of treatment). These subjects may more frequently use sterile needles for injections; therefore, the results of the current study are not generalizable to other populations. Nevertheless, HCV prevalence was higher in our studied group and thus, it is still recommended that training programs for addicted people and their families encourage the use of protective appliances (e.g., condoms or disposable syringes for injections) to prevent the outbreak of blood-borne and sexually-transmitted viral infections in this high-risk group.

\section{Acknowledgments}

The authors of this study take this opportunity to thank all patients, healthcare staff, and Rafsanjan MMT center directors for their kind assistance.

\section{References}

1. Napier TC, Vocci FJ. Addiction to alcohol and drugs of abuse is a horrific tragedy for affected individuals and their families, and a financial, legal, and social burden for societies. Pharmacol Ther. 2005;108(1):1-2. doi: 10.1016/j.pharmthera.2005.06.017. [PubMed: 16154202].

2. Rahimi-Movaghar A, Amin-Esmaeili M, Haghdoost AA, Sadeghirad B, Mohraz M. HIV prevalence amongst injecting drug users in Iran: a systematic review of studies conducted during the decade 1998-2007. Int J Drug Policy. 2012;23(4):271-8. doi: 10.1016/j.drugpo.2011.09.002. [PubMed: 22000694].

3. Perez Gonzalez K, Domingo-Salvany A, Hartnoll R. [Prevalence of human immunodeficiency virus and risk behaviours among opioid users seen in an emergency room]. Gac Sanit. 1999;13(1):7-15. [PubMed: 10217672].

4. Des Jarlais DC, Friedman SR. HIV infection among intravenous drug users: epidemiology and risk reduction. AIDS. 1987;1(2):67-76. [PubMed: 3130084].

5. Napoli C, Tafuri S, Pignataro N, Tedesco G, Maria AS, Quarto M. Risk factors for HBV/HIV/HCV in drug addicts: a survey of attendees of a department of pathological dependence. J Prev Med Hyg. 2010;51(3):1014. [PubMed: 21361113].

6. Caiaffa WT, Zocratto KF, Osimani ML, Martinez PL, Radulich G, Latorre L, et al. Hepatitis C virus among non-injecting cocaine users (NICUs) in South America: can injectors be a bridge?. Addiction. 2011;106(1):143-51. doi: 10.1111/j.1360-0443.2010.03118.x. [PubMed: 20955486].

7. McGovern MP, McLellan AT. The status of addiction treatment research with co-occurring substance use and psychiatric disorders. J Subst Abuse Treat. 2008;34(1):1-2. doi: 10.1016/j.jsat.2007.03.007. [PubMed: 17574797].

8. Zamani S, Kihara M, Gouya MM, Vazirian M, Nassirimanesh B, Ono-Kihara M, et al. High prevalence of HIV infection associated with incarceration among community-based injecting drug users in Tehran, Iran. J Acquir Immune Defic Syndr. 2006;42(3):342-6. doi: 10.1097/01.qai.0000219785.81163.67. [PubMed: 16639351].

9. Shekarchizadeh H, Ekhtiari H, Khami MR, Virtanen JI. Patterns of pretreatment drug abuse, drug treatment history and characteristics of addicts in methadone maintenance treatment in Iran. Harm Reduct J. 2012;9:18. doi: 10.1186/1477-7517-9-18. [PubMed: 22676557].

10. Moolchan ET, Umbricht A, Epstein D. Therapeutic drug monitoring in methadone maintenance: choosing a matrix. $J$ Addict Dis. 2001;20(2):55-73. doi:10.1300/J069v20n02_05. [PubMed:11318398].

11. Esteban J, de la Cruz Pellin M, Gimeno C, Barril J, Mora E, Gimenez $\mathrm{J}$, et al. Detection of clinical interactions between methadone and anti-retroviral compounds using an enantioselective capillary electrophoresis for methadone analysis. Toxicol Lett. 2004;151(1):243-9. doi:10.1016/j.toxlet.2004.04.007. [PubMed: 15177659].

12. Verster A, Buning E. Methadone guidelines. Amsterdam, Netherlands Euro-Methwork; 2000.

13. Golden J, O'Dwyer AM, Conroy RM. Depression and anxiety in patients with hepatitis C: prevalence, detection rates and risk factors. Gen Hosp Psychiatry. 2005;27(6):431-8. doi: 10.1016/j.genhosppsych.2005.06.006. [PubMed: 16271658]. 
14. Alavian SM, Tavallaei SA, Hoseini SM, Hedayati MR, Sepehrinia A. Prevalence of Depression in Chronic Hepatitis B and C Patients Treated with Interferon. Trauma Mon. 2007;12(2):161-7.

15. Alavian SM. Hepatitis $C$ infection in Iran; A review article. Arch Clin Infec Dis. 2009;4(1):47-59.

16. Haghdoost AA, Mostafavi E, Mirzazadeh A, Navadeh S, Feizzadeh A, Fahimfar N, et al. Modelling of HIV/AIDS in Iran up to 2014. J AIDS HIV Res. 2011;3(12):231-9. doi:10.5897/JAHR11.030.
17. Parvaresh N, Masoudi A, Majidi-Tabrizi S, Mazhari S. The Correlation between Methadone Dosage and Comorbid Psychiatric Disorders in Patients on Methadone Maintenance Treatment. Addict Health. 2012;4(1-2):1-8. [PubMed: 24494130].

18. Shirazi KK, Heydarnia E, Kamalianfard S, Heydarnia M, Fararooie M, Rahimi E. P01-104-Exploring the mental status of people with opium and heroin addiction. European Psychiatr. 2011;26(1):104. 